\section{(A) OPEN ACCESS}

- Additional material is published online only. To view please visit the journal online (http://dx.doi.org/10.1136/ ejhpharm-2012-000235).

${ }^{1}$ International Health Economics and Reimbursement, Amgen (Europe) GmbH, Zug, Switzerland ${ }^{2}$ Market Access, Amgen (Austria) GmbH, Vienna, Austria

${ }^{3} \mathrm{HEALTH}$-Institute for Biomedicine and Health Sciences, Joanneum Research Forschungsgesellschaft $\mathrm{mbH}$ Graz, Austria

${ }^{4}$ Health Economics and Reimbursement, Amgen AB, Solna, Sweden

${ }^{5}$ Institute of Pharmaceutical Medicine (ECPM), University of Basel, Basel, Switzerland ${ }^{6}$ Health Economics and Reimbursement, Amgen Switzerland AG, Zug, Switzerland.

\section{Correspondence to} Dr M Lothgren, International Health Economics and Reimbursement Amgen (Europe) $\mathrm{GmbH}$ Dammstrasse 23, Zug 6301, Switzerland; mickaell@amgen.com

Received 25 September 2012 Revised 8 January 2013 Accepted 18 January 2013 Published Online First 21 February 2013

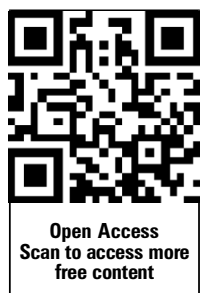

To cite: Lothgren $M_{\text {, }}$ Ribnicsek E, Schmidt L, et al. Eur I Hosp Pharm 2013;20:227-231.

\title{
Cost per patient and potential budget implications of denosumab compared with zoledronic acid in adults with bone metastases from solid tumours who are at risk of skeletal-related events: an analysis for Austria, Sweden and Switzerland
}

\author{
Mickael Lothgren, ${ }^{1}$ Erna Ribnicsek, ${ }^{2}$ Louise Schmidt, ${ }^{3}$ Wolfgang Habacher, ${ }^{3}$ \\ Jonas Lundkvist, ${ }^{4}$ Alena M Pfeil, ${ }_{1}^{5}$ Irina Biteeva, ${ }^{6}$ Polina Vrouchou, ${ }^{1}$ Andrea Bracco ${ }^{1}$
}

\section{ABSTRACT}

Objectives To assess cost implications per patient, per year, and to predict the potential annual budget impact when patients with bone metastases secondary to solid tumours at risk of skeletal-related events (SREs) transition from zoledronic acid (ZA; 4 mg every 3-4 weeks) to denosumab (120 mg every 4 weeks) in Austria, Sweden and Switzerland.

Methods Country specific costs for medication and administration, patient management and SREs (defined as pathologic fracture, radiation to bone, surgery to bone and spinal cord compression) were assessed over a 1-year time horizon. Drug administration and patient management costs were taken from available public sources. SRE costs were based on local unit costs applied to country specific healthcare resources obtained from a multinational retrospective chart review study. Due to lack of real world data for the included countries, SRE rates were derived from phase III clinical trials in patients with advanced cancer and bone metastases. These trials demonstrated that denosumab was superior to ZA in the reduction of SREs. Results Estimated total annual cost savings for each patient transitioned from ZA to denosumab varied by country and cancer type, ranging from $€ 1583$ to $€ 2375$ in Austria, from €1980 to €2319 in Sweden (9.1 SEK/€) and from $€ 3408$ to $€ 3857$ in Switzerland (1.2 CHF/€). Cost savings were mainly driven by the lower SRE related costs and lower administration costs of denosumab compared with ZA.

Conclusions Denosumab offers superior efficacy compared with ZA in patients with solid tumours and bone metastases. Cost savings are predicted in the Austrian, Swedish and Swiss healthcare systems following treatment transition from ZA to denosumab.

\section{INTRODUCTION}

Patients with advanced solid tumours commonly develop bone metastases. ${ }^{1}$ Bone metastases cause bone destruction through increased osteoclast activity, ${ }^{1}$ frequently resulting in skeletal complications known as skeletal-related events (SREs; commonly defined as pathologic fracture, radiation to bone, surgery to bone and spinal cord compression). SREs are associated with significant and debilitating pain, impaired morbidity, reduced quality of life, ${ }^{23}$ substantial health-resource utilisation ${ }^{4}$ and associated costs. ${ }^{5}$
Bisphosphonates are bone targeted agents that have been historically used to reduce the risk of SREs in patients with bone metastases. Zoledronic acid (ZA; Zometa ${ }^{\circledR}$, Novartis) has been considered the standard of care and has been shown to prolong time to first SRE and reduce the number of SREs; however, many patients with bone metastases continue to experience SREs. ZA is infused intravenously (IV) every 3-4 weeks and is associated with renal toxicity (necessitating monitoring of renal function prior to each infusion). ${ }^{6}$ Clinic visits for administration are, on average, 100 minutes in duration. ${ }^{7}$

Denosumab (Xgeva ${ }^{\circledR}$, Amgen Inc.) has a different and novel mode of action; it is the first fully human monoclonal IgG2 antibody that binds to RANK ligand. RANK ligand is an essential mediator of the formation, activation and survival of osteoclasts. ${ }^{1}$ Denosumab (120 mg subcutaneous injection (SC) every 4 weeks) was compared with ZA (4 mg IV every 4 weeks) in three identically designed phase III head to head clinical trials of patients with bone metastases from solid tumours (breast cancer, prostate cancer and other solid tumours or multiple myeloma (ClinicalTrials.gov: NCT00321464, NCT00321620 and NCT0033075)). In patients with solid tumours, denosumab was superior to ZA in reducing the risk of first on-study SREs and delaying the time to multiple (first and subsequent) SREs compared with ZA. Patients treated with denosumab also experienced fewer SREs overall than those treated with ZA. ${ }^{8-10}$ Denosumab does not require renal monitoring. The overall incidence of adverse events was similar between denosumab and ZA. ${ }^{8-10}$

The objective of the analyses reported in this paper was to assess the cost implications per patient, per year, and to predict the potential annual budget impact when patients with bone metastases secondary to solid tumours at risk of SREs transitioned from treatment with ZA (4 mg IV every 3-4 weeks) to treatment with denosumab (120 mg SC, every 4 weeks) in Austria, Sweden and Switzerland.

\section{METHODS}

\section{Design}

Analyses included country specific cost implications for medication, administration, patient management and SRE related costs per patient, per year, 
the resulting cost difference per patient, per year, and the prediction of potential annual budget impact with treatment transition from ZA to denosumab in patients with bone metastases from solid tumours. Real world data on the frequency of administration of ZA were used in the main analyses. The analyses included the following cost components: medication and administration, patient management and SRE related costs.

\section{Resource use and costs}

\section{Drug unit costs}

Drug unit costs were based on the available 2012 public prices in each of the countries (table 1); medication cost analyses were based on the drug unit costs and the frequency of administration of ZA and denosumab. The summary of product characteristics for ZA recommends administration every $3-4$ weeks, ${ }^{6}$ and evidence suggests that there is equivalent efficacy between these two administration schedules. ${ }^{11}$ For the purposes of these analyses, information on the frequency of ZA administration every 3 weeks in routine medical practice were obtained from a market research audit, the European Tandem Oncology Monitor (ETOM). ${ }^{12}$ The number of ZA administrations per year, per tumour type, were estimated based on the average number of patients receiving ZA every 3 weeks in Germany, Italy, Spain, the UK and France, due to the lack of such data for Austria, Sweden and Switzerland. The percentage of patients receiving ZA every 3 weeks was estimated to be $20.7 \%$ in breast cancer, $36.4 \%$ in prostate cancer and $29.1 \%$ in other solid tumours. This corresponds to 13.9, 14.6 and 14.3 doses per year of $\mathrm{ZA}$ in breast cancer, prostate cancer and other solid tumours, respectively. For denosumab, the approved administration schedule is once every 4 weeks, corresponding to 13 doses per year. ${ }^{13}$ In the base case analysis, the presented real world administration frequencies were used for ZA. Sensitivity analyses are presented for the scenario where both ZA and denosumab are administered every 4 weeks.

\section{Administration and patient management costs}

For Austria, unit costs were based on physicians' fee scales across provinces, ${ }^{14}$ outpatient tariffs set in one province for self-paying patients (as a proxy for outpatient costs), ${ }^{15}$ and national diagnosis and procedure related reimbursement rates for inpatients. ${ }^{16}$

For Sweden, base case drug administration costs were estimated from the mean of two hospital price lists that were judged to be representative of costs across the entire country. ${ }^{17} 18$

Table 1 Local unit costs by country

\begin{tabular}{lccc}
\hline & Austria & Sweden & Switzerland \\
\hline Drug and administration costs (per administration) (€) & & \\
$\quad$ Price denosumab 120 mg* & 371.00 & 354.40 & 452.46 \\
Price zoledronic acid 4 mg * & 303.10 & 308.79 & 336.71 \\
Administration cost—denosumab (SC) & 10.98 & 46.48 & 47.94 \\
Administration cost-zoledronic acid (IV) & 29.55 & 151.10 & 144.79 \\
Patient management costs (per test) (€) & & & \\
$\quad$ Laboratory test (serum creatinine test) & 7.46 & 3.08 & 2.10 \\
SRE costs (per SRE event) (€) & 10305 & 5802 & 25987 \\
$\quad$ Pathologic fracture & 14603 & 3305 & 13407 \\
Radiation to bone & 21496 & 10783 & 49330 \\
Surgery to bone & 22191 & 13143 & 51188 \\
$\quad$ Spinal cord compression & & & \\
\hline *Per 4 week cycle & & & \\
SRE, skeletal-related event. & &
\end{tabular}

For Switzerland, drug administration costs were based on publicly available tariff costs. Additional cost information was gathered using a doctors' survey to identify the TARMED ${ }^{19}$ items (outpatient tariff codes used in Switzerland) charged for IV and SC administration.

Patient management costs, including creatinine tests, were derived from publicly available sources in each of the countries. $^{14}$ 20-22

\section{SRE rates and costs}

In the absence of available real world SRE rates for the countries included in these analyses, annualised SRE rates for ZA were estimated for each tumour type, using the total number of SREs observed and total person years of follow-up in the above mentioned denosumab phase III clinical trials (table 2). ${ }^{8-10}$ Rates for denosumab were estimated by applying the rate ratios representing treatment effects (ie, times to first and subsequent SREs) of denosumab compared with ZA on the baseline ZA SRE rate.

The costs per SRE type for each country were generated by applying local unit costs applied to country specific SRE related healthcare resource use obtained from a multinational retrospective chart review study (20090146 study). ${ }^{5}$ This study enrolled patients with bone metastases or bone lesions secondary to breast cancer, prostate cancer, lung cancer or multiple myeloma from centres in Austria, the Czech Republic, Finland, Greece, Poland, Portugal, Sweden and Switzerland. Health-resource utilisation extracted from patient charts included inpatient stays, outpatient visits, day care visits, emergency room visits and procedures. Cost of SREs by SRE type for Austria and Sweden were derived from this study and have been reported previously, as listed in table $1 .^{5}$ For Switzerland, SRE costs were calculated slightly differently as a sum of inpatient visits, outpatient visits and outpatient procedure costs (table 1). ${ }^{23} 24$ In order to estimate outpatient visit costs, additional information was obtained from a physicians' survey among physicians aimed at identifying the typical outpatient tariff $\left(\right.$ TARMED ${ }^{19}$ ) codes charged for outpatient consultations. Average costs were estimated based on Swiss Cantonal costs per TARMED point. ${ }^{19}{ }^{25}$ Mean number of inpatient stays and health-resource utilisation estimates for the number and duration of inpatient visits were taken from the 20090146 study. A weighted average of adjusted length of stay was calculated based on hospitalisation statistics of the Organisation for Economic Cooperation and Development. Data

Table 2 Skeletal-related event rate and distribution by type

\begin{tabular}{lccc}
\hline & $\begin{array}{c}\text { Breast } \\
\text { cancer }\end{array}$ & $\begin{array}{l}\text { Prostate } \\
\text { cancer }\end{array}$ & $\begin{array}{c}\text { Other solid } \\
\text { tumours }\end{array}$ \\
\hline $\begin{array}{l}\text { SRE rate (per year) } \\
\quad \text { Zoledronic acid }\end{array}$ & 0.631 & 0.947 & 0.936 \\
$\quad \begin{array}{l}\text { Denosumab (derived) } \\
\text { Denosumab treatment effect }\end{array}$ & 0.486 & 0.777 & 0.796 \\
$\quad$ Rate ratio first and subsequent SRE & 0.77 & 0.82 & 0.85 \\
$\quad$ SRE type distribution* (\%) & & & \\
$\quad$ Pathologic fracture & 58.2 & 26.8 & 31.4 \\
$\quad$ Radiation to bone & 35.4 & 66.1 & 57.5 \\
$\quad$ Surgery to bone & 4.7 & 1.5 & 6.2 \\
$\quad$ Spinal cord compression & 1.7 & 5.6 & 5.0 \\
\hline *Pooled across both treatment groups. & & & \\
$\quad$ SRE, skeletal-related event. & & & \\
& &
\end{tabular}


from the Swiss National Statistics Office were used to calculate the cost of hospital stays. ${ }^{26}$

Costs are reported in Euros $(€)$. Swedish and Swiss costs were converted to Euros using exchange rates of $9.1 \mathrm{SEK} / €$ and 1.20 $\mathrm{CHF} / €$, respectively.

\section{RESULTS}

Estimates of cost implications per patient, per year, included the costs of drugs and drug associated patient management (creatinine test) costs, administration and SRE costs, as shown in table 3.

In all countries, treatment transition from ZA to denosumab (using real world data on the frequency of administration of ZA) was predicted to generate cost savings (table 3 ). The estimated total annual cost savings per patient transitioned from treatment with ZA to denosumab varied by country and tumour type and ranged from $€ 1583$ to $€ 2375$ in Austria, from $€ 1980$ to $€ 2319$ in Sweden and from $€ 3408$ to $€ 3857$ in Switzerland. The cost savings were mainly driven by the delay in the time to the first and first and subsequent SREs, the lower SRE related costs and administration costs of denosumab compared with ZA. Given the higher SRE related costs estimated for Austria and Switzerland compared with Sweden, reductions in SRE related costs had a greater impact in Austria and Switzerland. The administration cost savings were significantly higher in Sweden and Switzerland compared with Austria, due to the unit cost difference associated with IV and SC administration.

The total annual budget implications per country for patients that transition treatment from ZA to denosumab depends on the actual transition rate across the different tumour types. Figure 1 illustrates the cost saving predictions following treatment transition from ZA to denosumab for up to 1500 patients for each tumour type in Austria, Sweden and Switzerland. Based on these findings the predicted total annual cost savings for every 1000 patients transitioned from ZA to denosumab ranged from $€ 1.6$ to $€ 2.4$ million in Austria, from $€ 2.0$ to $€ 2.3$ million in Sweden and from $€ 3.4$ to $€ 3.6$ million in Switzerland.

Sensitivity analyses were conducted for cost related inputs (administration and SRE related costs), trial based data on the frequency of administration of ZA (every 4 weeks) and baseline $\mathrm{ZA}$ risk (ZA SRE rate). Administration and SRE related costs varied by $\pm 50 \%$. The baseline ZA SRE rate for patients receiving ZA varied by $+100 \%$ and $-50 \%$ based on the results of a recent US study indicating that the real world SRE rate may be substantially higher (up to twofold) than the trial based rates used in the current analyses. ${ }^{27}$ These sensitivity analyses confirmed the robustness of the results (see online supplementary appendix tables A1-3).

In addition to the above sensitivity analyses, price threshold analyses were conducted to assess the impact of expected price reductions for ZA following generic formulation entry due to loss of patent protection in the middle of 2013. The threshold analyses present the maximum price reduction for ZA that will still maintain the overall cost saving results of denosumab given the currently available prices for ZA (see online supplementary appendix table A4). If the prices for ZA in comparison with the current prices as presented in table 1 are reduced by more than the threshold value, denosumab may no longer be cost saving. The results from the ZA price threshold analyses reveal a very strong cost savings impact of denosumab, even with significant price reductions for $\mathrm{ZA}$ in all three countries. Denosumab remains cost saving even with price reductions for $\mathrm{ZA}$ of up to $38-54 \%$ in Austria, $46-52 \%$ in Sweden and $71-79 \%$ in Switzerland.

If the ZA price reduction is greater than the threshold value, treatment transition to denosumab will increase costs associated with treatment. In this situation, a cost effectiveness analysis would be in order, where the incremental costs for denosumab could be contrasted against the improved patient health related quality of life obtained as a consequence of the clinical

Table 3 Cost implications per patient per year by cost category, tumour type and country

\begin{tabular}{|c|c|c|c|c|c|c|}
\hline & \multicolumn{2}{|l|}{ Breast cancer } & \multicolumn{2}{|c|}{ Prostate cancer } & \multicolumn{2}{|c|}{ Other solid tumours } \\
\hline & Denosumab & Zoledronic acid* & Denosumab & Zoledronic acid* & Denosumab & Zoledronic acid* \\
\hline \multicolumn{7}{|l|}{ Austria } \\
\hline Cost of drugs & 4823 & 4212 & 4823 & 4418 & 4823 & 4323 \\
\hline Cost of administration/management & 143 & 514 & 143 & 540 & 143 & 528 \\
\hline Cost of SREs & 6100 & 7922 & 10856 & 13239 & 11198 & 13174 \\
\hline Total costs & 11066 & 12648 & 15821 & 18196 & 16164 & 18024 \\
\hline Total cost difference (denosumab vs ZA) & \multicolumn{2}{|c|}{$-1583(12.5 \%)$} & \multicolumn{2}{|c|}{$-2375(13.1 \%)$} & \multicolumn{2}{|c|}{$-1861(10.3 \%)$} \\
\hline \multicolumn{7}{|l|}{ Sweden } \\
\hline Cost of drugs & 4607 & 4291 & 4607 & 4501 & 4607 & 4404 \\
\hline Cost of administration/management & 604 & 2143 & 604 & 2248 & 604 & 2199 \\
\hline Cost of SREs & 2564 & 3330 & 3601 & 4392 & 4016 & 4725 \\
\hline Total costs & 7775 & 9764 & 8812 & 11140 & 9228 & 11327 \\
\hline Total cost difference (denosumab vs ZA) & \multicolumn{2}{|c|}{$-1988(20.4 \%)$} & \multicolumn{2}{|c|}{$-2328(20.9 \%)$} & \multicolumn{2}{|c|}{$-2100(18.5 \%)$} \\
\hline \multicolumn{7}{|l|}{ Switzerland } \\
\hline Cost of drugs & 5882 & 4679 & 5882 & 4908 & 5882 & 4802 \\
\hline Cost of administration/management & 623 & 2041 & 623 & 2141 & 623 & 2095 \\
\hline Cost of SREs & 11204 & 14550 & 15091 & 18403 & 17095 & 20112 \\
\hline Total costs & 17709 & 21271 & 21596 & 25453 & 23600 & 27008 \\
\hline Total cost difference (denosumab vs ZA) & \multicolumn{2}{|c|}{$-3562(16.7 \%)$} & \multicolumn{2}{|c|}{$-3857(15.2 \%)$} & \multicolumn{2}{|c|}{$-3408(12.6 \%)$} \\
\hline
\end{tabular}



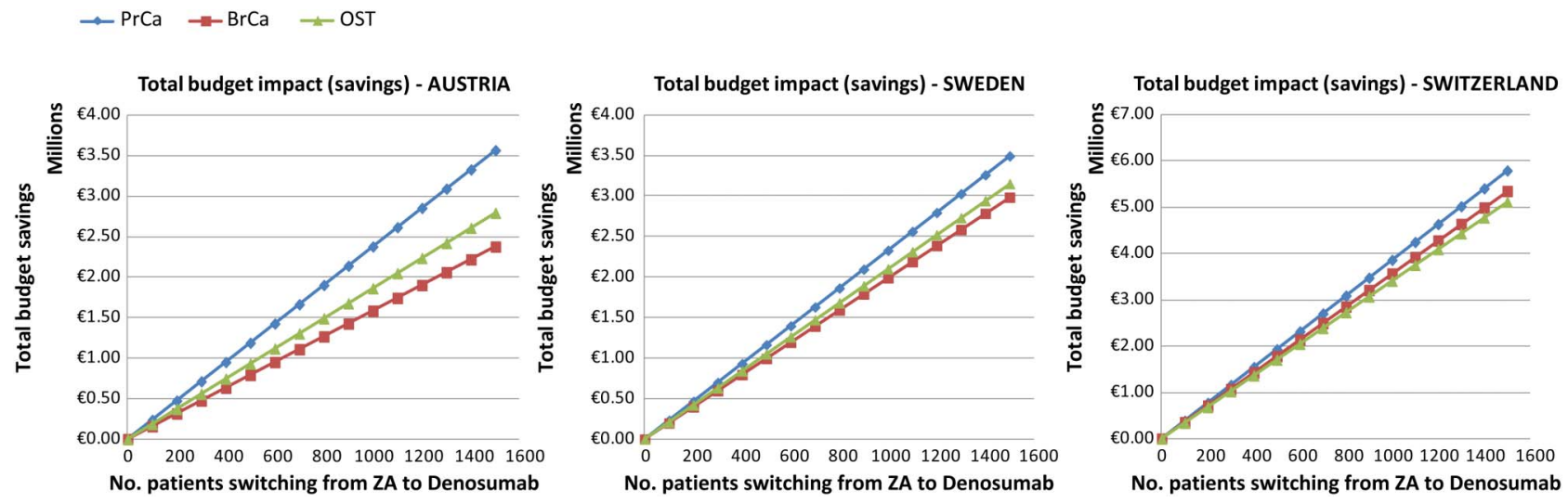

Figure 1 Predicted total budget implications following treatment transition from zoledronic acid (ZA) to denosumab per country and tumour type. BrCa, breast cancer; OST, other solid tumours; PrCa, prostate cancer.

superiority of denosumab. Ultimately, the decision makers' willingness to pay for improvements in health and treatment outcomes will determine this cost effectiveness assessment. A complete cost effectiveness assessment was beyond the scope of this analysis, and the threshold analyses indicated that denosumab will remain cost saving even when accounting for significant price reductions in ZA.

\section{DISCUSSION}

Budget impact analysis is a tool for payers at national and regional levels and contributes to informed decision making on the most efficient allocation of healthcare resources. Decisions on the usage of innovative drugs can have a significant impact on patients' health and outcomes. The purpose of this study was to assess the cost implications per patient, per year, the resulting cost difference per patient, per year, and to predict the potential annual total budget impact implications of treatment transition from ZA to denosumab in adults with bone metastases from solid tumours at risk of SREs in the Austrian, Swedish and Swiss healthcare settings.

Across the three countries, treatment transition from ZA to denosumab resulted in substantial cost savings per patient, per year. Denosumab was associated with lower overall SRE related costs due to its clinical superiority over ZA. Additionally, the administration costs of denosumab (administered SC) were lower than those for ZA (infused IV). Sensitivity analyses confirmed the robustness of the results.

One of the main limitations of these analyses was the use of trial based SRE rates due to a lack of real world SRE data in Austria, Sweden and Switzerland. According to the results of a recent study in the USA, the real world SRE rate may be substantially higher (up to twofold) than the trial based rates used in these analyses. ${ }^{27}$ Hence these analyses might have underestimated the annualised SRE rates and the value of denosumab. Additionally, some economic benefits to the wider society (eg, a reduction in indirect costs associated with SREs and treatment administration) and the impact on caregivers were not included in this analysis.

Another potential limitation is that the real world data used for the actual frequency of administration of ZA (every 3 weeks vs every 4 weeks) were not based on country specific data for Austria, Sweden and Switzerland as these were not available. Instead, the average frequency of ZA use every 3 weeks that was used in these analyses was estimated from market research data (ETOM) for Germany, Italy, Spain, the UK and France.
Furthermore, the analyses were performed using a 1 year time horizon and therefore do not represent the total benefits in terms of cost per patient benefit of denosumab, given that most patients live longer than 1 year.

Finally, as the analyses focused solely on the potential budget implications with treatment transition from ZA to denosumab they did not incorporate the additional clinical benefits observed in the relevant clinical trials associated with denosumab, in terms of improved patient reported outcomes, including health related quality of life and superior delay in pain progression. ${ }^{28}$

\section{CONCLUSION}

Denosumab is predicted to offer a cost saving treatment option compared with ZA, with improved clinical outcomes in terms of reduced risk of SREs. Therefore, it represents good value for money in preventing the risk of SREs in patients with bone metastases from solid tumours in Austria, Sweden and Switzerland.

\section{Key messages}

Denosumab offers superior efficacy compared with zoledronic acid in patients with solid tumours and bone metastases.

- Cost savings are predicted in the Austrian, Swedish and Swiss healthcare systems following treatment transition from zoledronic acid to denosumab.

Acknowledgements Emma Thomas revised the draft manuscript and Sarah Petrig provided editorial support.

Contributors $M L, A B$ and PV contributed to the design of this modelling study, the analysis plan, analysed the data, and drafted, revised and approved the final publication. ML is guarantor for these data. ER, LS and WH provided data, application of the model and interpretation from an Austrian perspective, as well as drafting, revising and approving the final publication. JL provided data, application of the model and interpretation from a Swedish perspective, as well as drafting, revising and approving the final publication. AMP and IB provided data, application of the model and interpretation from a Swiss perspective, as well as drafting, revising and approving the final publication.

Funding This research was funded by Amgen (Europe) $\mathrm{GmbH}$.

Competing interests $M L, E R, J L, I B, P V$ and $A B$ are employees of Amgen. $L S$ and WH are employees of Joanneum Research Forschungsgesellschaft $\mathrm{mbH}$, which received funding from Amgen to conduct this research. AMP has received research funding for the University of Basel from Amgen.

Provenance and peer review Not commissioned; externally peer reviewed.

Open Access This is an Open Access article distributed in accordance with the Creative Commons Attribution Non Commercial (CC BY-NC 3.0) license, which permits others to 
distribute, remix, adapt, build upon this work non-commercially, and license their derivative works on different terms, provided the original work is properly cited and the use is non-commercial. See: http://creativecommons.org/licenses/by-nc/3.0/

\section{REFERENCES}

1 Roodman GD. Mechanisms of bone metastasis. N Engl J Med 2004:350:1655-4.

2 Rubens RD. Bone metastases-the clinical problem. Eur J Cancer 1998;34:210-13.

3 Weinfurt KP, Li Y, Castel LD, et al. The significance of skeletal-related events for the health-related quality of life of patients with metastatic prostate cancer. Ann Oncol 2005; 16:579-84.

4 Bahl A, Hoefeler H, Duran I, et al. Health resource utilisation (HRU) associated with skeletal-related events (SRES) in patients with bone metastases (BM): tesults of a prospective multinational observational study. J Clin Oncol 2011;29;15(Suppl): e16523.

5 Gunther O, Body JJ, Sleeboom H, et al. Cost of skeletal complications from bone metastases: results from a retrospective European Study. Support Care Cancer 2012.

6 European Medicines Agency. Zometa (zoledronic acid): summary of product characteristics. 2012;20:589.

7 Oglesby A, Sherif B, Odom D, et al. Time and costs associated with preparing and administering zoledronic acid in patients with breast or prostate cancer and metastatic bone disease. Commun Oncol 2009;6:494-502.

8 Stopeck AT, Lipton A, Body JJ, et al. Denosumab compared with zoledronic acid for the treatment of bone metastases in patients with advanced breast cancer: a randomized, double-blind study. J Clin Oncol 2010;28:5132-9.

9 Fizazi K, Carducci M, Smith M, et al. Denosumab versus zoledronic acid for treatment of bone metastases in men with castration-resistant prostate cancer: a randomised, double-blind study. Lancet 2011;377:813-22.

10 Henry D, Von Moos R, Hungria V, et al. Delaying skeletal-related events in a randomized phase III study of denosumab versus zoledronic acid in patients with advanced cancer. J Clin Oncol 2010;28(7s):abstract 9133.

11 Kretzschmar A, Wiege T, Al-Batran SE, et al. Rapid and sustained influence of intravenous zoledronic Acid on course of pain and analgesics consumption in patients with cancer with bone metastases: a multicenter open-label study over 1 year. Support Cancer Ther 2007:4:203-10.
12 Monitor ETO. Electronic Tandem Oncology Monitor 2012.

13 European Medicines Agency. Denosumab (XGEVA) Summary of Product Characteristics. 2012.

14 Österreichische Sozialversicherung. Services und Informationen für Vertragspartner. 2012. http://www.sozialversicherung.at/portal27/portal/esvportal/channel content/ cmsWindow? action=2\&p_menuid=2989\&p_tabid=5 (accessed 1 Sep 2012).

15 Das Land Steiermark Landesgesetzblatt. 2009.

16 Bundesministerium für Gesundheit, Leistungsorientierte Krankenanstaltfinanzierungs (LKF) Modell 2011.

17 Akademiska hospital Uppsala. Price list for Medical care, 2010.

18 Price list Southeast Health Region. Price List, 2011.

19 TARMED. TarmedSuisse Online browser 2012. http://onb.tarmedsuisse.ch/ (accessed 12 March 2012).

20 BAG Analysenliste. 2012. http://www.bag.admin.ch/themen/krankenversicherung/ 00263/00264/04185/index.html?lang=de (accessed 12 March 2012).

21 Landesgesetzblatt DLS. Das Land Steiermark Landesgesetzblatt. 2009; LGBI Stück 14:(Nr 45 und 6 p.).

22 County council of Gävle. Price list. Clinical chemistry and transfusion medicine, 2010.

23 Santèsuisse. Taxpunktwerte Ärzte. 2011. http://www.santesuisse.ch/de/dyn_output html? content.void=47323\&SID=453aa58313aca45c6df830ca457da269\&navid=753 (accessed 12 March 2012).

24 Delmore G, Biteeva I, Steinmann K, et al. Direct medical costs associated with skeletal-related events in Switzerland. Presented at ECHE. 2012.

25 Spezialitätenliste (SL) http://bag.e-mediat.net/SL2007.Web.External/ (accessed 12 March 2012).

26 Bundesamt für Statistik BFS. Krankenhausstatistik. Standarttabellen 2009. http://www.bfs.admin.ch/bfs/portal/de/index/themen/14/03/01/data/01.html (accessed 12 March 2012)

27 Hatoum HT, Lin SJ, Smith MR, et al. Zoledronic acid and skeletal complications in patients with solid tumors and bone metastases: analysis of a national medical claims database. Cancer 2008;113:1438-5.

28 Cleeland CS, Patrick D, Fallowfield L, et al. Effects of Denosumab vs Zoledronic acid on pain in patients with advanced cancer and bone metastases: an integrated analysis of 3 Pivotal Trials. Ann Oncol 2010;21(Suppl. 8):viii380 (1248P). 